International Journal of Pure and Applied Mathematics

Volume 93 No. 2 2014, 275-284

ISSN: 1311-8080 (printed version); ISSN: 1314-3395 (on-line version)

url: http://www.ijpam.eu

doi: http://dx.doi.org/10.12732/ijpam.v93i2.11

ijpam.eu

\title{
CYLINDRICALLY SYMMETRIC COSMIC STRINGS COUPLED WITH MAXWELL FIELDS IN BIMETRIC RELATIVITY
}

\author{
P.K. Sahoo ${ }^{1}$, B. Mishra ${ }^{2}$ \\ ${ }^{1,2}$ Department of Mathematics \\ Birla Institute of Technology and Science-Pilani \\ Hyderabad Campus \\ Hyderabad, 500078, INDIA
}

\begin{abstract}
Cylindrically symmetric cosmological models are obtained in Rosen's (1973) bimetric theory, when the source of gravitation is electromagnetic field coupled with one dimensional cosmic string. Some physical and kinematical properties of the model are discussed.
\end{abstract}

AMS Subject Classification: $83 \mathrm{D} 05$

Key Words: electromagnetic field, cosmic strings, bimetric theory

\section{Introduction}

Many authors have investigated the plane symmetric solutions of EinsteinMaxwell equations in general relativity. Here, we study the line element of a cylindrically symmetric metric in alternative theory of gravitation, i.e. Bimetric Theory of Relativity proposed by Rosen (1973). This theory agrees with the present observational facts pertaining to general relativity. In this theory, there are two metric tensors at each point of space-time- $g_{i j}$ which describes gravitation and the background metric $\gamma_{i j}$, which enters into the field equations and interacts with $g_{i j}$ but does not interact directly with matter. Accordingly, at every point of space-time point, one has two line elements

Received: March 3, 2014

(c) 2014 Academic Publications, Ltd.

$\S$ Correspondence author url: www.acadpubl.eu 


$$
\begin{aligned}
& d s^{2}=g_{i j} d x^{i} d x^{j} \\
& d \sigma^{2}=\gamma_{i j} d x^{i} d x^{j}
\end{aligned}
$$

The field equations of Rosen's bimetric theory of gravitation are

$$
N_{j}^{i}-\frac{1}{2} N \delta_{j}^{i}=-8 \pi \kappa T_{j}^{i}
$$

where

$$
N_{j}^{i}=\frac{1}{2} \gamma_{b}^{a}\left(g^{h i} g_{h j \mid a}\right)_{\mid b}
$$

and

$$
N=N_{i}^{i}, g=\operatorname{det}\left(g_{i j}\right), \gamma=\operatorname{det}\left(\gamma_{i j}\right), \kappa=\sqrt{ }\left(\frac{g}{\gamma}\right)
$$

A vertical bar $(\mid)$ denotes the covariant differentiation with respect to $\gamma_{i j}$ and $T_{j}^{i}$ is the energy momentum tensor of the matter fields.

The purpose of Rosen's bimetric theory is to get rid of the singularities that occur in general relativity and appear in the big-bang cosmological models. Therefore, there has been lot of interest in cosmological models related to Rosen's bimetric theory of gravitation. Several aspects of the bimetric theory of gravitation have been studied and investigated by Yilmaz (1975), Israelit (1979, 1981), Reddy and Venkateswarlu (1989), Mohanty et al. (2002), Sahoo (2009, 2010). Recently Sahoo and Mishra (2013) have studied axially symmetric space-time with strange quark matter attached to string cloud in bimetric theory.

The general relativistic treatment of strings was started by Letelier (1979, 1983) and Satchel (1980). They have given the energy momentum tensor for string distribution in the form

$$
T_{j(\text { strings })}^{i}=\rho u^{i} u_{j}-\lambda x^{i} x_{j}
$$

where

$$
u^{i} u_{i}=1=-x^{i} x_{i} \quad \text { and } \quad u^{i} x_{i}=0
$$

where $\rho$ is the rest energy density for a cloud with particles attached to them, $\lambda$ is the tension density. The unit time like vector $u^{i}$ is the flow vector and the unit space like vector $x^{i}$ specifies the direction of the string.The particle density associated with the configuration is given by

$$
\rho_{p}=\rho-\lambda
$$

Using these concepts, a number of general relativistic exact solutions were investigated, Krori et al. (1990) investigated Bianchi type II, $V I_{0}$, VIII and IX string 
cosmological models. Letelier (1979) has obtained the solution to Einstein field equations for cloud of string with spherical, plane, cylindrical symmetric spacetime. Nevin (1991) has solved Einstein's field equations for spherical or static cylindrical symmetry with string dust source. Baysal et al. (2001) have studied string cosmological models in cylindrical symmetric inhomogeneous universe in general relativity and various physical and geometrical properties of the model have been discussed. Mohanty and Sahoo (2003) have studied cylindrical symmetric space time with perfect fluid and scalar meson field in bimetric theory. Nonexistence of spherical symmetric cosmological model with perfect fluid and scalar filed coupled with cosmic string in bimetric theory has been shown by Sahoo (2008).

The magnetic field has important role at the cosmological scale and is present in galactic and intergalactic spaces. The importance of the magnetic field for various astrophysical phenomenon's has been studied in many papers. Melvin (1975) has pointed out that during the evolution of the universe, the matter is highly ionized state and is smoothly coupled with the field, subsequently forming neutral matter as a result of universe expansion. Therefore considering the presence of magnetic field in strings universe is not unrealistic and has been investigated by many authors [Banerjee et al. (1990), Shri Ram and Singh (1995), Singh and Singh (1999), Bali and Upadhaya (2003)]. Herrera and Leon (1985) have obtained exact analytic solution of the static EinsteinMaxwell equation for perfect and anisotropic fluid under the assumption of spherical symmetry and the existence of the one-parameter group of conformal motion. Static and spherical symmetric solution are found in bimetric theory for perfect anisotropic charged fluids by Khandalkar and Gawande (2010). Sahoo et al. (2011) have studied Bianchi type $\mathrm{V}$ and $V I_{0}$ cosmic strings coupled with Maxwell fields in bimetric theory. Katore et al. (2011) have investigated Bianchi type I massive string cosmological model for perfect fluid distribution in the presence of magnetic filed in bimetric relativity.

There is a lot of interest in alternative theories of gravitation, due to some lacunas in general relativity. In view of the importance of Maxwell's electromagnetic field interaction with cosmic string, we have discussed cylindrically symmetric cosmic string magnetized cosmological model in Rosen's bimetric theory of gravitation. The magnetic field is due to an electric current produced along the $\mathrm{x}$-axis with infinite electrical conductivity. 


\section{Metric and Energy Momentum Tensor}

The general cylindrical symmetric metric is considered in the form given by Marder (1958) as

$$
d s^{2}=A^{2}\left(d t^{2}-d x^{2}\right)-B^{2} d y^{2}-C^{2} d z^{2}
$$

where $A, B$ and $C$ are functions of $x$ and $t$.

In order to find a specific solution, we confine ourselves to the form of metric given by Singh and Singh (1969) as

$$
d s^{2}=A^{2}\left(d t^{2}-d x^{2}\right)-B^{2} d y^{2}-C^{2} d z^{2}
$$

where $A, B$ and $C$ are functions of time $t$ alone.

The metric represents the anisotropic homogeneous universe [Aygun et al. (2007)].

The background flat metric corresponding to (8) is

$$
d \sigma^{2}=d t^{2}-d x^{2}-d y^{2}-d z^{2}
$$

The energy momentum tensor for a cosmic cloud strings coupled with electromagnetic field is given by

$$
T_{i j}=T_{j(\text { strings })}^{i}+E_{j(m a g)}^{i}
$$

In the co-moving coordinate system, taking the string in radial direction, equation (4) takes the form

$$
T_{1}^{1}=\lambda ; \quad T_{2}^{2}=T_{3}^{3}=0 ; \quad T_{4}^{4}=\rho ; \quad T_{j}^{i}=0 \quad i \neq j
$$

The quantities $\rho$ and $\lambda$ are functions of $t$ only.

The electromagnetic tensor is described by

$$
E_{j}^{i}=-F^{i l} F_{j l}+\frac{1}{4} \delta_{j}^{i} F^{k l} F_{k l}
$$

Since the electromagnetic field along x-axis, the only non-vanishing components of $F_{i j}$ is $F_{23}=-F_{32}$ and due to the assumption of infinite electrical conductivity $F_{14}=F_{24}=F_{34}=0$.

The Maxwell's equation for the magnetic field

$$
F_{[i j, k]}=F_{i j, k}+F_{j k, i}+F_{k i, j}=0
$$


leads to $F_{23}=\frac{H B C}{A}$, where $H$ is constant.

Now from (12), we obtain the components of the electromagnetic field

$$
E_{1}^{1}=-E_{2}^{2}=-E_{3}^{3}=E_{4}^{4}=\frac{H^{2}}{2 A^{2}}=\eta(\text { say })
$$

Thus (10) leads to

$$
T_{1}^{1}=\lambda+\eta ; \quad T_{2}^{2}=-\eta=T_{3}^{3} ; \quad T_{4}^{4}=\rho+\eta
$$

\section{Field Equations in Bimetric Theory}

Rosen's bimetric field equations (3) for the cylindrically symmetric metric (8) with the help of (9) and (15) can be written as

$$
\begin{gathered}
\left(\frac{B_{4}}{B}\right)_{4}+\left(\frac{C_{4}}{C}\right)_{4}=16 \pi \kappa(\lambda+\eta) \\
2\left(\frac{A_{4}}{A}\right)_{4}-\left(\frac{B_{4}}{B}\right)_{4}+\left(\frac{C_{4}}{C}\right)_{4}=-16 \pi \kappa \eta \\
2\left(\frac{A_{4}}{A}\right)_{4}+\left(\frac{B_{4}}{B}\right)_{4}-\left(\frac{C_{4}}{C}\right)_{4}=-16 \pi \kappa \eta \\
\left(\frac{B_{4}}{B}\right)_{4}+\left(\frac{C_{4}}{C}\right)_{4}=16 \pi \kappa(\rho+\eta)
\end{gathered}
$$

where, suffix 4 indicates differentiation with respect to $t$.

From (17) and (18), we get

$$
\left(\frac{B_{4}}{B}\right)_{4}=\left(\frac{C_{4}}{C}\right)_{4}
$$

Hence the field equations (16)-(19) reduce to

$$
\begin{gathered}
\left(\frac{C_{4}}{C}\right)_{4}=8 \pi \kappa(\lambda+\eta) \\
\left(\frac{A_{4}}{A}\right)_{4}=-8 \pi \kappa \eta
\end{gathered}
$$




$$
\left(\frac{C_{4}}{C}\right)_{4}=8 \pi \kappa(\rho+\eta)
$$

From (21) and (23), we get

$$
\rho=\lambda(\text { Geometric string })
$$

Case 1: Geometric string cosmological model $(\eta=0)$. Since we have less field equations having more unknowns, if we consider $\eta=0$, then from (22), we obtain

$$
A=\exp \left(a_{1} t+a_{2}\right)
$$

From (21) and (23), we get

$$
\left(\frac{C_{4}}{C}\right)_{4}=8 \pi \kappa \lambda=8 \pi \kappa \rho
$$

So, finally we have a single equation involving two unknowns, hence to get a physically meaningful and singularity free solution as required by Rosen's theory, let us consider

$$
C=\left(a_{3} t+a_{4}\right)^{n} \exp \left(a_{3} t+a_{4}\right)
$$

then

$$
B=\left(a_{3} t+a_{4}\right)^{n} \exp \left(a_{5} t+a_{6}\right)
$$

and

$$
8 \pi \kappa \lambda=8 \pi \kappa \rho=\frac{n a_{3}^{2}}{\left(a_{3} t+a_{4}\right)^{2}}
$$

where $n$ is an arbitrary constant and $a_{i}$ 's are constants of integration.

The corresponding cosmological model can be written as

$$
\begin{aligned}
d s^{2}=\exp \left[2\left(a_{1} t+a_{2}\right)\right] & \left(d t^{2}-d x^{2}\right)-\left(a_{3} t+a_{4}\right)^{2 n} \\
\times & \left.\times \exp \left[2\left(a_{5} t+a_{6}\right)\right] d y^{2}+\exp \left[2\left(a_{3} t+a_{4}\right)\right] d z^{2}\right\} .
\end{aligned}
$$

Thus (30) together with (29) constitutes cylindrically symmetric geometric string cosmological model in bimetric theory. If $n=0$ the above model reduces to vacuum model [Adhav et al. (2010)].

Case 2: Magnetized cosmological model $(\eta \neq 0)$. Since the number of equations are less than the number of unknowns, to get a physically meaningful and singularity free solution, let us take

$$
C=\cosh \left(a_{7} t+a_{8}\right)
$$


Then from (20), we get

$$
B=\cosh \left(a_{7} t+a_{8}\right) \exp \left(a_{9} t+a_{10}\right)
$$

From (22), we have

$$
A=a_{13} \sinh \left(a_{11} t+a_{12}\right)
$$

where, $a_{13}=\frac{\sqrt{ } \overline{4 \pi \kappa} H}{a_{11}}$

From (14), we get

$$
\eta=\frac{a_{11}^{2}}{8 \pi \kappa} \operatorname{cosech}^{2}\left(a_{11} t+a_{12}\right)
$$

From (23), we have

$$
\lambda=\rho=\frac{a_{7}^{2} \operatorname{sech}^{2}\left(a_{7} t+a_{8}\right)+a_{11}^{2} \operatorname{cosech}^{2}\left(a_{11} t+a_{12}\right)}{8 \pi \kappa}
$$

The corresponding cosmological model can be written as

$$
\begin{aligned}
d s^{2}=a_{13}^{2} \sinh ^{2}\left(a_{11} t+\right. & \left.a_{12}\right)\left(d t^{2}-d x^{2}\right) \\
& -\cosh ^{2}\left(a_{7} t+a_{8}\right)\left\{\exp \left[2\left(a_{9} t+a_{10}\right)\right] d y^{2}+d z^{2}\right\} .
\end{aligned}
$$

\section{Physical and Geometrical Properties}

The volume element of the geometric string cosmological model (30) is given by

$$
V=\sqrt{-g}=\left(a_{3} t+a_{4}\right)^{2 n} \exp \left(a_{14} t+a_{15}\right)
$$

where $a_{14}=2 a_{1}+a_{3}+a_{5}$ and $a_{15}=2 a_{2}+a_{4}+a_{6}$. Since $n$ is an arbitrary constant it may be positive, negative or zero. In bimetric theory the model does not have singularity. Hence $n$ should be non-negative. If $n$ is positive or zero, the volume increase as time increases. The model (30) is expanding with time and has no singularity.

The volume element of the magnetized cosmological model (36) is given by

$$
V=\sqrt{-g}=a_{13}^{2} \sinh ^{2}\left(a_{11} t+a_{12}\right) \cosh ^{2}\left(a_{7} t+a_{8}\right) \exp \left(a_{9} t+a_{10}\right)
$$

The spatial volume for the model (36) increases with time.

The expansion scalar $\theta$ and the shear scalar $\sigma^{2}$ for the geometric string model (30) are given by

$$
\theta=a_{14}+\frac{2 n a_{3}}{a_{3} t+a_{4}}
$$




$$
\sigma^{2}=\frac{1}{6}\left[a_{14}+\frac{2 n a_{3}}{a_{3} t+a_{4}}\right]^{2}
$$

It can be seen that for large $t$ the values of $\theta$ and $\sigma^{2}$ becomes constant if $n \neq 0$. In case of $n=0, \theta$ and $\sigma^{2}$ are independent of time.

The expansion scalar $\theta$ and the shear scalar $\sigma^{2}$ for the geometric string model (36)are given by

$$
\begin{gathered}
\theta=2 a_{11} \operatorname{coth}\left(a_{11} t+a_{12}\right)+2 a_{7} \tanh \left(a_{7} t+a_{8}\right)+a_{9} \\
\sigma^{2}=\frac{7}{18}\left[2 a_{11} \operatorname{coth}\left(a_{11} t+a_{12}\right)+2 a_{7} \tanh \left(a_{7} t+a_{8}\right)+a_{9}\right]^{2}
\end{gathered}
$$

Here we can observe that for the model (36), the expansion scalar and the shear scalar are increasing with time.

The deceleration parameter for geometric string cosmological model (30) is given by

$$
q=\frac{6 n a_{3}^{2}\left(a_{3} t+a_{4}\right)}{\left\{a_{14}\left(a_{3} t+a_{4}\right)+2 n a_{3}\right\}^{2} \times \exp \left(a_{1} t+a_{2}\right)}-1
$$

The deceleration parameter for magnetized cosmological model (36) is given by

$$
\begin{aligned}
q= & -[1+ \\
& \left.\frac{6 a_{11}^{2} \operatorname{cosech}^{2}\left(a_{11} t+a_{12}\right)+6 a_{7}^{2} \operatorname{sech}^{2}\left(a_{7} t+a_{8}\right)}{\left\{2 a_{11} \operatorname{coth}\left(a_{11} t+a_{12}\right)+2 a_{7} \tanh \left(a_{7} t+a_{8}\right)+a_{9}\right\}^{2} \times a_{13} \sinh \left(a_{11} t+a_{12}\right)}\right] .
\end{aligned}
$$

We can observe that for both the models the deceleration parameter $q$ is less than zero for large values of $t$. Hence, the models represent accelerating universe.

\section{Conclusion}

In this paper, the field equations for anisotropic cylindrically symmetric metric are solved in bimetric theory of gravitation when the energy momentum tensor is one dimensional cosmic cloud string coupled with electromagnetic field. The models (30) and (36) will play a significant role in the study of cosmic strings coupled with electromagnetic field in bimetric theory of gravitation. It is observed that the absence of magnetized field we get geometric string cosmological model. The cosmological models presented here are free from singularities. 


\section{Acknowledgment}

PKS acknowledges UGC, New Delhi, India for financial assistance to carry out the Minor Research Project [No. F. 41-1385/2012(SR)].

\section{References}

[1] N. Rosen: Gen. Relativ. Gravit., 4 (1973), 435.

[2] H. Yilmaz: Gen. Relativ. Gravit., 6 (1975), 269.

[3] M. Israelit: Gen. Relativ. Gravit.,11 (1979), 25.

[4] M. Israelit: Gen. Relativ. Gravit.,13 (1981), 681.

[5] D.R.K. Reddy and R. Venkateswarlu: Astrophys. Space Sci., 158 (1989), 169 .

[6] G. Mohanty,P.K. Sahoo and B. Mishra: Astrophys. Space Sci.,281 (2002), 609.

[7] P.K. Sahoo:Int. J. Theo. Phys., 48 (2009), 2022.

[8] P.K. Sahoo:Int. J. Theor. Phys.,49 (2010), 25.

[9] P.K. Sahoo and B. Mishra: Int. J. of Pure and Appl. Math.,82(1) (2013), 87.

[10] P.S. Letelier: Phys. Rev. D20 (1979), 1249.

[11] P.S. Letelier: Phys. Rev. D28 (1983), 2414.

[12] J. Satchel:Phys. Rev. D, 21 (1980), 2171.

[13] K.D. Krori, T. Chaudhury, C.R.Mahanta and A. Mazumdar, : Gen. Relativ. Gravit.,22 (1990),123.

[14] J.M. Nevin: Gen. Relativ. Gravit.,23 (1991), 253.

[15] H. Baysal, I.Yavuz, I. Tarhan, U. Camci and I. Yilmaz: Turk. J. Phys.,25 (2001), 283.

[16] G. Mohanty and P.K. Sahoo: Comm. Phys., 13(3)(2003), 137. 
[17] P.K. Sahoo:Int. J. Theor. Phys.,47 (2008), 3029.

[18] M.A. Melvin:Ann. New York Acad. Sci. ,262 (1975), 253.

[19] P.A. Banerjee, A.K. Sanyal and S. Chakraborthy:Pramana J. Phys.,34 (1990), 1.

[20] Shri Ram and T.K. Singh:Gen. Relativ. Gravit.,27 (1995), 1207.

[21] G.P. Singh and T.K. Singh:Gen. Relativ. Gravit.,31 (1999), 371.

[22] R. Bali and R.D. Upadhaya: Astrophys. Space Sci.,283 (2003), 97.

[23] L. Herrera and J. Ponce de Leon:J. Math. Phys.,26 (1985), 778.

[24] S. P. Khandalkar and S.P. Gawande: Astrophys. Space Sci.,326 (2010), 45.

[25] P.K. Sahoo, B. Mishra and A. Ramu:Int. J. Theor. Phys.,50 (2011), 349.

[26] S.D. Katore, R.S. Rane and K. S. Wankhade:Pramana J. Phys.,76 (2011), 543.

[27] L. Marder: Proc. R. Soc. London,A246 (1958), 133.

[28] K.P. Singh and D.N. Singh:J. Phys. A (general Phys.),2 (1969), 28.

[29] S. Aygun and I. Tarhan: Pramana J. Phys.,68 (2007), 21.

[30] K.S. Adhav, A.S. Nimkar, U.R.Ugale and R. S. Thakare: Adv. Stud. Theor. Phys., 4 (2010), 917. 